BULLETIN OF THE

AMERICAN MATHEMATICAL SOCIETY

Volume 80, Number 4, July 1974

\title{
A MEROMORPHIC FUNCTION WITH ASSIGNED NEVANLINNA DEFICIENCIES
}

\author{
BY DAVID DRASIN ${ }^{1}$
}

Communicated by F. W. Gehring, October 17, 1973

\section{Statement of result.}

THEOREM. Let $\delta(a)$ and $\theta(a)$ be nonnegative functions defined on the extended complex plane $\hat{C}$ such that $0 \leqq \delta(a)+\theta(a) \leqq 1, a \in \hat{C}$,

$$
\sum_{a \in C}\{\delta(a)+\theta(a)\} \leqq 2 .
$$

Then there exists a function $f(z)$ which is meromorphic in the finite $z$-plane with $\delta(a, f)=\delta(a), \theta(a, f)=\theta(a), a \in \hat{C}$. Finally, let $\phi(r)$ be $a$ positive increasing function with

$$
\phi(r) \rightarrow \infty \quad(r \rightarrow \infty) .
$$

Then our function $f(z)$ may be chosen so that, in addition, its Nevanlinna characteristic satisfies

$$
T(r)<r^{\phi(r)}
$$

for all sufficiently large $r$.

Here we are using the standard notations of Nevanlinna's theory as described in [3], [6]; for example, $\theta(a, f)$ is the index of multiplicity (Verzweigungsindex) of $a$. Our function $f(z)$ thus provides a complete solution to the 'inverse problem' of the Nevanlinna theory (cf. [2, Chapter 7]; [9, Chapter 8]).

In general, the solution to the inverse problem must be of infinite order (cf. [8]); (1.2) asserts that this may be as 'small' an infinite order as desired.

Among earlier partial solutions to this problem we note Nevanlinna [5], Goldberg (cf. [2, Chapter 7, Theorems 8.2, 8.3]) and Fuchs-Hayman (cf. $[3, \S 4.1])$.

2. Method of proof. Given the function $\phi(r)$ of (1.1) and the sets $\Delta=\{a ; \delta(a)>0\}$ and $\theta=\{a ; \theta(a)>0\}$, we shall associate a sequence $\left\{r_{k}\right\}$

AMS (MOS) subject classifications (1970). Primary 30A70.

${ }^{1}$ Research partially supported by National Science Foundation, and performed while on sabbatical leave at Imperial College, London.

Copyright @ American Mathematical Society 1974 
with $r_{k+1} / r_{k}$ tending rapidly to infinity with the property that if $b$ is a fixed element of $\hat{C}-(\Delta \cup \theta)$ then, for all $a \in \hat{C}$,

$$
\begin{aligned}
\mid\{1-\delta(a)\} n(r, b, f)- & n(r, a, f) \mid \\
& \leqq \\
(4 / k) n(r, b, f) & \left(r_{k} \leqq r \leqq r_{k+1}\right), \\
|\theta(a) n(r, b, f)-\{n(r, a, f)-\bar{n}(r, a, f)\}| & \\
& <(4 / k) n(r, b, f) \quad\left(r_{k} \leqq r \leqq r_{k+1}\right)
\end{aligned}
$$

and

$$
\begin{aligned}
2^{k}\left(1-k^{-1}\right) & \leqq n(2 r, b, f) / n(r, b, f) \\
& \leqq 2^{k+1}\left(1+k^{-1}\right) \quad\left(r_{k} \leqq r \leqq r_{k+1}\right) .
\end{aligned}
$$

That $f$ has preassigned deficiencies and indices of multiplicity follows from (2.1), (2.2) and the fact (cf. [6, p. 276]) that there is a set $E$ of logarithmic capacity zero such that

$$
N(r, a, f) \sim T(r, f) \quad(r \rightarrow \infty, a \notin E) .
$$

Also, if $r_{k+1} / r_{k}$ increases sufficiently rapidly, (2.3) shows that the growth of $T(r, f)$ may be retarded in accord with (1.2).

One first constructs a 'quasi-meromorphic' function ${ }^{2} g(z)$ which satisfies, formally, (2.1), (2.2) and (2.3), and then factors

$$
g=f \circ h
$$

where $f$ is a meromorphic function and $h$ is a quasiconformal homeomorphism of the complex plane onto itself. The problem is to ensure that $h$ in (2.4) sufficiently approximates the identity so that (2.1), (2.2) and (2.3) (with, perhaps, a different sequence $\left\{\boldsymbol{r}_{k}\right\}$ ) transfer to $f$.

Using an important principle of Teichmuller [7], Le Van Thiem [4] first applied this principle to the inverse problem, and the method was further exploited by Goldberg (cf. [2, Chapter 7]). These efforts had two limitations: the characteristic of $g$ had to be of finite order and the dilatation of $g, d_{g}(z)=\left|g_{\bar{z}}(z) / g_{z}(z)\right|$ was subject to

$$
\iint_{|z| \geqq 1} d_{g}(z)|z|^{-2} d x d y<\infty .
$$

In $[1$, Theorem 2], it was shown that this principle applies under the more flexible condition

$$
D_{g}(r) \equiv \int_{0}^{2 \pi} d_{g}\left(r e^{i \theta}\right) d \theta=o(1) \quad(r \rightarrow \infty),
$$

\footnotetext{
2 A 'quasi-meromorphic function' is one which may be expressed as in (2.4).
} 
and the freedom allowed by (2.6) is decisive here. For it is not hard to show that $f$ and $g$ will have the same deficiencies and indices of multiplicity if $D_{g}(r)$ decreases very rapidly with respect to $\log \{n(2 r, b, g) / n(r, b, g)\}$. By increasing the ratios $r_{k+1} / r_{k}$ we can diminish $D_{g}(r)$ with respect to $\log \{n(2 r, b, g) / n(r, b, g)\}$; thus $(2.5)$ is very unlikely to hold. Finally, $g$ is constructed by piecing together functions discussed in [1] and [5].

ADDED IN PROOF (MARCH 15, 1974). Dr. A. A. Goldberg has informed me that the substitution of (2.6) for (2.5) first appears in the work of P. P. Belinskii. The behavior of quasiconformal mappings at an isolated singular point, Učen. Zap. L'vov. Gos. Univ. 29 (1954), 58-70. (Russian). However, Belinskii did not apply this to the inverse problem.

\section{REFERENCES}

1. D. Drasin and A. Weitsman, Meromorphic functions with large deficiency sum, Advances in Math. (to appear).

2. A. A. Gol'dberg and I. V. Ostrovskil, Distribution of values of meromorphic functions, "Nauka", Moscow, 1970; English transl., Transl. Math. Monographs, Amer. Math. Soc., Providence, R.I. (to appear).

3. W. K. Hayman, Meromorphic functions, Oxford Mathematical Monographs, Clarendon Press, Oxford, 1964. MR 29 \#1337

4. Le-Van Thiem, Uber das Umkehrproblem der Wertverteilungslehre, Comment. Math. Helv. 23 (1949), 26-49. MR 11, 22.

5. R. Nevanlinna, Über Riemannsche Flächen mit endlich vielen Windungspunkten, Acta Math. 58 (1932), 295-373.

6. - Eindeutige analytische funktionen, 2 nd ed., Die Grundlehren der math. Wissenschaften, Band 46, Springer-Verlag, Berlin, 1953; English transl., Die Grundlehren der math. Wissenschaften, Band 162, Springer-Verlag, Berlin and New York, 1970. MR 15, 208; 43 \#5003.

7. O. Teichmuller, Untersuchungen über konforme und quasikonforme Abbildung, Deutsche Math. 3 (1938), 621-678.

8. A. Weitsman, $A$ theorem on Nevanlinna deficiencies, Acta Math. 128 (1972), 41-51.

9. H. Wittich, Neuere Untersuchungen über eindeutige analytische Funktionen, Springer, Berlin, 1955. MR 17, 1067.

Department of Mathematics, Purdue University, West Lafayette, Indiana 47907 